\title{
Computational Investigation of the NASA Cascade Cyclonic Separation Device
}

\author{
Nathaniel C. Hoyt ${ }^{1}$, Yasuhiro Kamotani ${ }^{2}$, Jaikrishnan Kadambi ${ }^{3}$ \\ Case Western Reserve University, Cleveland, OH, 44106 \\ John B. McQuillen ${ }^{4}$, John M. Sankovic ${ }^{5}$ \\ NASA Glenn Research Center, Cleveland, OH, 44135
}

\begin{abstract}
Devices designed to replace the absent buoyancy separation mechanism within a microgravity environment are of considerable interest to NASA as the functionality of many spacecraft systems are dependent on the proper sequestration of interpenetrating gas and liquid phases. Inasmuch, a full multifluid Euler-Euler computational fluid dynamics investigation has been undertaken to evaluate the performance characteristics of one such device, the Cascade Cyclonic Separator, across a full range of inlet volumetric quality with combined volumetric injection rates varying from $1 \mathrm{~L} / \mathrm{min}$ to $20 \mathrm{~L} / \mathrm{min}$. These simulations have delimited the general modes of operation of this class of devices and have proven able to describe the complicated vortex structure and induced pressure gradients that arise. The computational work has furthermore been utilized to analyze design modifications that enhance the overall performance of these devices. The promising results indicate that proper CFD modeling may be successfully used as a tool for microgravity separator design.
\end{abstract}

\section{Nomenclature}

$\begin{array}{ll}A & =\text { area } \\ \alpha_{i} & =\text { volume fraction of the } i^{\text {th }} \text { phase } \\ \beta_{i} & =\text { volumetric quality of the } i^{\text {th }} \text { phase } \\ d_{\text {sauter }} & =\text { Sauter mean diameter } \\ D & =\text { dispersed phase bubble/droplet diameter } \\ \varepsilon_{\text {underflow }} & =\text { underflow separation efficacy } \\ \varepsilon_{\text {overflow }} & =\text { overflow separation efficacy } \\ j_{i} & =\text { volumetric flux of the } i^{\text {th }} \text { phase }\end{array}$

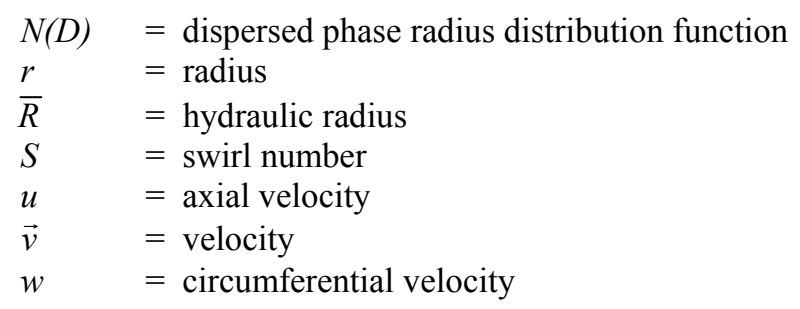

\section{Introduction}

$\mathrm{P}$ hase separation, particularly for gas-liquid flows, is a multiphase flow consideration that acquires great importance in physical domains lacking the presence of any appreciable gravitational acceleration. While bubble removal is a naturally occurring phenomenon for most terrestrial considerations, the absence of the buoyancyinduced agency in a microgravity environment often results in situations wherein two disparate phases have no distinct inclination to secede from one another. This can present a number of problems with respect to the functioning of many spacecraft and space station systems ranging from turbomachinery to Environmental Control and Life Support Systems. ${ }^{1}$

As such, many phase separator devices have been constructed and investigated to facilitate this absent separation mechanism. Devices affording phase separation without the requirement of moving mechanical

\footnotetext{
${ }^{1}$ Graduate Researcher, Mechanical and Aerospace Engineering, Student Member

${ }^{2}$ Professor, Mechanical and Aerospace Engineering, Associate Fellow

${ }^{3}$ Professor, Mechanical and Aerospace Engineering, Non-member

${ }_{5}^{4}$ Aerospace Engineer, Fluid Physics and Transport Branch, Non-member

${ }^{5}$ Supervisory Biomedical Engineer, Fluid Physics and Transport Branch, Non-member
} 
components or exceptional power input are classified as being passive and have been examined closely owing to their dependability and simplicity. ${ }^{1-3}$

A common passive separation approach, and that which is the focus of the current study, employs the inertia of the multiphase flow itself to provide the desired phase-parting action. The conceptual basis of these devices involves the creation of a swirling flow by tangential injection of a multiphase fluid stream into the separator device. This eccentric injection thereby creates a buoyancy-like separation action via the pressure gradient that arises to maintain the necessary centripetal acceleration of the fluid as it swirls within the device housing.

This family of devices, typically called cyclonic separators, has been in development for terrestrial industrial purposes for decades. The Cascade Cyclonic Separation Device (CSD-C) is a microgravity specific example of this separator design philosophy that has been in development at Glenn Research Center under McQuillen, et. al since the mid-1990's. ${ }^{4}$ The below schematic diagrams depict the geometry of this device. Table 1 indicates the dimensions of the relevant geometric parameters.

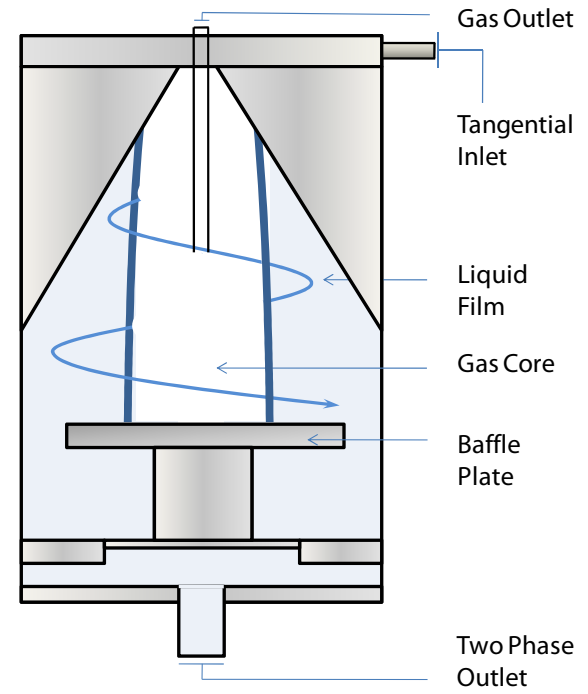

Figure 1: CSD-C Separator Geometry

In these designs the two phase mixture is injected at a small radius to facilitate high initial separatory action while a baffle plate positioned within the flow itself is used to arrest and reverse the axial flow of the less dense phase that has been directed to the center of the device by the induced radial pressure gradient. The increased residence time of this phase within the swirling flow region assists the completion of the separation process. Gas is hence removed through the overflow outlet extending into the center of the device while the dense liquid is removed from the bottom. This volume of gas not only ensures that the gas outlet remains unencumbered by liquid but has also already been observed to act a much-needed sink or source for the outlet in the event of temporary variability of the gas input. ${ }^{5}$

While experimental apparatuses of the CSD-C have, at minimum, shown efficacy for certain specified input conditions, ${ }^{6}$ failures with offdesign conditions (such as non-optimal flow rates or with mist development, etc.) and the unknown delimitation of what constitutes "off-design" dictates that the flow characteristics of this device be more fully investigated. ${ }^{7}$ The data gathered in reduced gravity flights thus far have mainly included separation efficiency metrics, differential pressure information, and videos of the gas core development, leaving full velocity profiles as yet unascertained. As any improvement of the device design requires more information regarding the flow characteristics within, a thorough computational investigation of the CSD-C has been performed.

Toward that end, a consistent, systematic computational fluid dynamics simulation procedure was developed such that accurate simulations of the flow within the CSD-C could be achieved. The results thereof were then employed to further elucidate the phenomenological details of these complicated vortex flows and to improve possible future design iterations of the device.

\section{Modeling Approach}

The CFD models employed were based upon the Fluent commercial finite volume solver coupled with as needed userdefined modifications. The description of the multiphase character of the flow required special attention. Any Euler-Lagrange approach would be incapable of describing the complete range of expected volume fractions encountered within a properly functioning separation device, while interfacial tracking methods (such as VOF or front-tracking) would be too computationally expensive to simulate a bubbly flow of this complexity. Inasmuch, a complete Euler-Euler multifluid approach was employed as doing so requires no assumption of a limited volume loading. The method, in general, treats the flow as that of two interpenetrating phases (with respective momentum and continuity equations) that are coupled through interphase momentum exchange and a common pressure. ${ }^{8}$

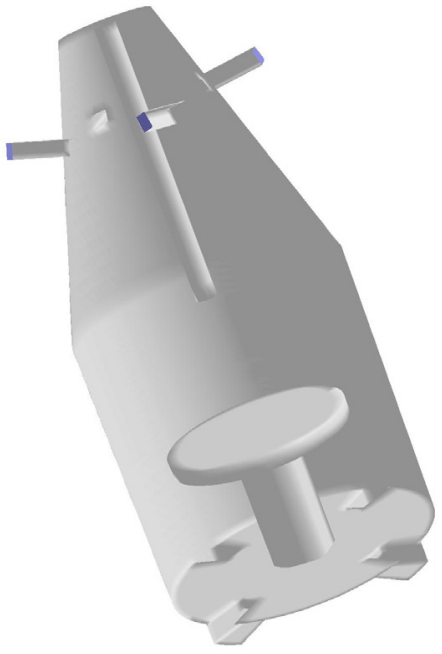

Figure 2: Exposed Rendered Geometry 
The interphase momentum exchange, which is primarily a result of the drag between the respective phases, was prescribed using a bubble/droplet drag term modified by both the local volume fraction and the local turbulence length scale in a manner akin to Lane et. al. ${ }^{9}$ A symmetric approach with respect to the volume fraction was utilized to account for the regions of the flow where the phase primality may be exchanged between the respective phases. ${ }^{8}$ This symmetric approach in tandem with extended drag correlations such as those of Ishii and Zuber, and Gidaspow $^{10}$ allow for a reasonable description of the drag terms for volume fractions tending toward either pure liquid or pure gas regimes (roughly to $0.0 \leq \alpha \leq 0.3$ and $0.85 \leq \alpha$ $\leq 1.0$ ). Application of these correlations to intermediate

Table 1. Baseline CSD-C Relevant Dimensions

\begin{tabular}{ll}
\hline \hline Inlet Area [x4], in $^{2}$ & 0.0425 \\
Cylinder Radius, in & 2.5 \\
Cylinder Length, in & 4.5 \\
Overflow Outlet Radius, in & 0.125 \\
Overflow Outlet Depth, in & 4.5 \\
Baffle Plate Radius, in & 1.5 \\
Baffle Plate Depth, in & 0.25 \\
Underflow Outlet Area [x4], in ${ }^{2}$ & 0.0785 \\
Frustum Angle & $24^{\circ}$ \\
\hline \hline
\end{tabular}
volume loadings is questionable, but the computational cells possessing $\alpha$ in this range are generally few in number (owing to the separator's intended operation); inasmuch, the incurred inconsistencies are presumed to be somewhat limited. Further complicating the interphase momentum exchange term is the specification of a bubble/droplet diameter with respect to the complicated coalescence/breakup and fission/fusion phenomena that occur. A Sauter mean diameter, $d_{\text {sauter }}$ can be specified to characterize the local behavior of a distribution of bubbles $/$ droplets, ${ }^{11}$ but the evolution of this parameter throughout the separator via a coalescence kernel is, in general, limited to dispersed volume loadings. ${ }^{12}$ Specifically,

$$
d_{\text {sauter }}=\int_{0}^{\infty} D^{3} N(D) d D / \int_{0}^{\infty} D^{2} N(D) d D
$$

In absence of any available applicable correlations, the coalescence and breakup phenomena were left uncharacterized, and the disperse-phase bubble/droplet radii were specified only through a constant Sauter mean diameter consistent with experimental observation.

The mixture level turbulence field too deserved special attention as the common first-order $k-\varepsilon$ and $k-\omega$ closure models are generally incapable of describing the extra rates of strain associated with large streamline curvature. ${ }^{13}$ Special modifications to the $k-\varepsilon$ formulation (such as the renormalization group approach) are typically prescribed to work for moderate swirl cases such that the swirl number $S$ is less than 0.5 , but cannot sufficiently describe the anisotropy present for more highly swirling flows. ${ }^{8}$ The swirl number is a metric characterizing the degree to which a flow is swirling and is defined as

$$
S=\int r w \vec{v} \cdot d \vec{A} /\left(\bar{R} \int u \vec{v} \cdot d \vec{A}\right)
$$

As results would later indicate, the swirl number for a nominal CSD-C configuration can reach values with magnitudes as high as $\mathrm{O}\left(10^{1}\right)$, hence necessitating the use of complete Reynolds Stress transport modeling. Turbulence modification due to the presence of the secondary phase could be taken into account in disperse regions of the flow utilizing a turbulence length scale modification, ${ }^{14}$ but complete applicability to the entire range of volume loading was, again, limited.

The gas core interface is yet another modeling issue that must be afforded special attention when using a multifluid modeling description of the flow. Of particular concern are spurious velocities (so-called parasitic velocities) that are created at an interface due to inconsistencies in the common pressure field. ${ }^{15}$ The body-forceweighted pressure field (i.e. the dominant radial pressure gradients resulting from the vortex flow) and the relative simplicity of the gas core shape specific to the CSD-C, however, allow for resolution of these inconsistencies. To accomplish this, interfacial identification and correction through the imposition of local momentum sources within the equations of motion for the respective phases was utilized. The use of momentum source terms to account for general surface tension effects, however, cannot be easily implemented a priori; ${ }^{15}$ furthermore, the resolution of the mesh was typically insufficient to resolve the normal vector and curvature of the interface in the lateral directions. Hence, capillarity effects related to the core/film were not taken into account.

The multifluid approach incurs further assumptions insofar as the injected two-phase stream was necessarily presumed to possess a bubbly/droplet character. As the multiphase flow pattern in simple tube geometries in a reduced gravity environment can be experimentally observed to deviate greatly from bubbly conditions for even 
somewhat modest volume loading (bubbly flows have been found to transition to slug flows within small tubes for $\alpha$ as little as 0.45 , and within large tubes for as little as 0.20$),{ }^{16}$ this multifluid approach can be seen to be deficient with respect to accounting for the unknown flow patterns that may develop at the two-phase injection ports. The flow within the separator body itself could too deviate greatly from any assumed bubbly specification (particularly with regards to the aforementioned specification of a fixed Sauter mean diameter). Full resolution of these problems remains unresolved. The current modeling methods employed, however, are expected to account for the salient flow features within phase separation devices (specifically the discernment of the separation mechanism and the vortex flow features).

While similar CFD methodologies have been used to model terrestrial separators, it is believed that this is the first time they have been utilized to describe microgravity gas-liquid separation devices. Validation of the approach was not directly achieved for the microgravity device but was instead performed using available experimental data taken from a roughly analogous terrestrial separator device studied by Hsieh, et al. ${ }^{17}$ These comparisons clearly demonstrated the need for second-order closure for the turbulence models. ${ }^{18}$ Interfacial identification and correction of the core was also found to provide some improvement in the prediction of the flow split parameters, but the elimination of the parasitic velocities was typically not crucial in the highly swirling flows due to the overwhelmingly swirling character of the flow. With the consistency of the approach thus confirmed, the methods were applied to the microgravity separation device.

The boundary conditions utilized for the CSD simulations consisted of the imposition of bubbly/droplet flow at the inlets. This two-phase injection was specified at the velocity inlets via input of both a total volumetric flux $j_{\text {tot }}$ (the sum of the superficial component velocities) and a gas volumetric quality $\beta_{\text {gas,inlet, }}$ where

$$
\begin{aligned}
& j_{\text {tot }}=\sum_{i} j_{i}=\sum_{i} \alpha_{i} u_{i}=\left(1-\alpha_{\text {gas }}\right) u_{\text {liquid }}+\alpha_{\text {gas }} u_{\text {gas }} \\
& \beta_{\text {gas, inlet }}=j_{\text {gas }} / j_{\text {tot }}
\end{aligned}
$$

Typically, the phase velocities were specified to be equal, thereby making the $\beta_{\text {gas, inlet }}$ parameter tantamount to an inlet volume fraction. Volumetric flow rates studied ranged roughly from $1 \mathrm{~L} / \mathrm{min}$ to $20 \mathrm{~L} / \mathrm{min}$ across the entire range of volume fractions (keeping in mind the modeling limitations and caveats inherent to the various flow domains across this range). High liquid rates were indeed considered even though the device was originally intended to be operated solely in a cascade mode. ${ }^{1}$ Subsequent to implementation, a complete grid sensitivity study was performed in order to ensure the proper convergence of the employed numerical descriptions. Complete details of the implementation can be found in Ref. 19.

\section{Results}

The results of the CFD study delimited many flow features that are impossible to discern from the current experimental data for the CSD-C.

\section{A. General Flow Characteristics}

Examination of the general flow characteristics allowed insight into the generation of the separatory action. Plots of the angular velocity along the device radius for typical conditions showed that the placement of the inlets, as intended, promoted quick initial separation of the gas from the liquid as a result of the high centripetal accelerations afforded by the distinct free and forced vortex regions. These velocities (which are the source of the separation pressure gradients) are plotted in Fig. 3 for a typical set of boundary conditions $\left(j_{\text {inlel }}=3.0 \mathrm{~m} / \mathrm{s}, \beta_{\text {inlet }}=0.3\right)$. The corresponding axial velocity profiles are also included in Fig. 3. Near the top of the device (interrogation line 1), high velocity peaks near the wall result from the outward confinement of the liquid layer as it is injected with slight obliquity into the frustum section. The velocities rise again toward the axis of the device due to the upward angular momentum transport provided by a recirculating flow region. Toward the center of the device (interrogation lines 2 and 3), the tangential velocity profiles develop a combined free/forced vortex character; however, the velocity peaks at the free/forced vortex junction are not as pronounced as those for typical terrestrial particulate separators as the inward transport of momentum is now primarily that of a diffusion process (the typically predominant inward advective process being largely eliminated by the presence of the gas core). Examination of the axial velocities shows that the careful placement of the baffle plate causes the aforementioned recirculation region; in doing so, the bubbles are redirected upward to again experience the strong separation gradients provided toward the top of the device. Gas core visualization corresponding to these injection parameters is provided by Fig. $5 \mathrm{c}$ of the next section. 

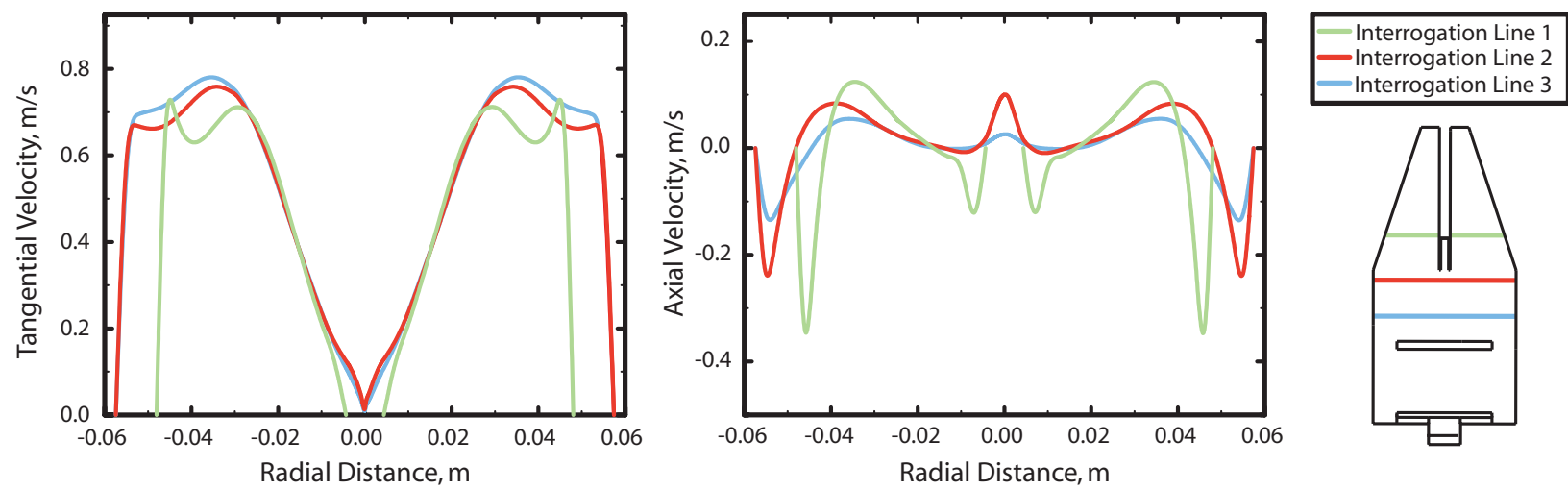

Figure 3. Mixture Tangential Velocities At Various Interrogation Lines

\section{B. Modes of Operation}

Beyond simple calculation of the internal flow fields, the CFD study has served to elucidate the various modes under which the CSD-C can be found to operate. The first mode of operation is that of a semi-homogeneous flow wherein the separation pressure gradients are insufficient to disentangle the respective phases (Fig. 4). This corresponds to an off-design condition such that sufficient slip velocities required to completely separate the respective phases from one another are not generated. In the absence of this relative motion, the fluid fields essentially act as a coupled entity. A necessary flow condition leading to this state of operation is that of a high liquid volumetric quality, as otherwise the symmetric Stokes number specification in general ensures negligible interphase momentum exchange when the gas exists locally as the dominant continuum phase (excluding the possibility of misty flows). Beyond this, further requirements on the flow conditions include low superficial velocities (thereby eliminating the cyclonic pressure gradient), high turbulence intensity (thereby augmenting both dispersion effects and the coefficient of drag), or a very small Sauter diameter of the injected bubbles (thereby facilitating large interphase momentum exchange). The small radius of the separator's injection ports, however, typically assure that sufficient initial separatory action is created for all but the most degenerate flow conditions. The evolution of this semi-homogeneous condition into a fully inhomogeneous one where the buoyancy action is sufficient to separate the respective phases cannot be described with confidence due to the uncertainty implicit in the correlations used to describe the interphase momentum terms.

Beyond these low flow velocities where the separation action is insufficient, the CFD methodologies employed made various modes of inhomogeneous operation discernable. These remaining modes of operation are all inhomogeneous in that separation (at least initially) is able to occur. Immediate separation at the injection ports owing to combined centripetal, wall adhesion, and deflector-plate effects, however, does not necessarily ensure proper separator function at either outlet. Three typical flow patterns arise from these conditions. The diverse behavior for given sets of inlet conditions was found to arise as dictated by an outlet mass-flow induced pressure accommodation mechanism and an gas core pressure matching contraction/expansion mechanism. Three general

inhomogeneous modes of operation, as classified by the form of the gas core, are shown in Fig. 5. While each of these figures show a mode that initially separates the liquid/gas mixture from one another, the global sequestration of the two respective phases can be clearly seen to be dependent upon more than this initial separation alone (as, in Fig. 5c, for example, the gas outlet is adulterated by liquid).

The first of these inhomogeneous states is that of the adhered film (Fig. 5a). Occurring at high gas injection rates, this regime is typified by the presence of a thin liquid layer around the periphery of the separator device. The multifluid CFD approach utilized in this study is least suited to this particular mode (which is unfortunate as it is the intended mode of operation for the cascade device), but meaningful results can still be generated.

Along with the direct swirl induced effects, the gas-liquid separation in this mode is induced in large part by a deflector plate-like mechanism wherein the wall acts essentially as an oblique flow obstruction. The deflection occurs as the finite breadth of the inlets prevents the injected fluid from being entirely of tangential character. Highly localized pressure gradients arise at the initial injection point into the separator. This initial effect can be amplified (if so desired) by redirecting the inlets radially inward. Such a modification sacrifices a component of the tangential velocity (hence reducing the angular momentum injection rate) in exchange for increased obliquity of the impinging flow. 
Table 2. Film Centripetal Acceleration Adhered Film: Baseline CSD-C

\begin{tabular}{ccc}
\hline \hline $\begin{array}{c}\text { Total Volumetric } \\
\text { Flow Rate }(\mathrm{L} / \mathrm{min})\end{array}$ & $\begin{array}{c}\text { Volumetric } \\
\text { Quality }\end{array}$ & $\begin{array}{c}\text { Film Centripetal } \\
\text { Acceleration ("g") }\end{array}$ \\
\hline 19.4 & 0.875 & 0.105 \\
9.7 & 0.875 & 0.02 \\
4.85 & 0.875 & 0.0057 \\
\hline \hline
\end{tabular}

Angular momentum conservation necessitates a quick loss of tangential velocity for the fluid film as it proceeds down the expansion frustum. The inducement of this film to the wall is, as such, reduced significantly. Table 2 shows values for the effective film centripetal acceleration at the separator midplane for a selected set of differing total volumetric fluxes.

Not surprisingly, the accelerations clearly possess a velocity-squared dependency. At this location half way down the axis of the device, these accelerations serve only to maintain the sequestration of the respective phases that has been previously facilitated at the injection zone. The small magnitude of these accelerations is related directly to the confinement of the liquid flow to the wall and the associated loss of the vortex character of the flow. As external accelerations encountered in a reduced gravity experiment (such as those conducted via a parabolic flight plan) are generally of order $0.01 \mathrm{~g}$, the continued sequestration of the fluid film is put somewhat into question for low superficial velocities. The subsequent modes of operation (corresponding to on-design conditions) possess extended separation gradients of order $1 \mathrm{~g}$ and beyond that well exceed any temporal accelerations encountered in the reduced gravity experimentation.

A primary deficiency of the employed modeling approach with respect to this mode of operation is the absence of wall adhesion. This free energy reduction that is afforded by the presence of the liquid would serve to keep the film in place in spite of the extraneous body forces. The very small thickness of the film is too a problem as the liquid layer easily retreats to within the wall adjacent cells (where non-equilibrium wall functions persist).

While the purity of the overflow is maintained in this flow regime, the liquid film at the underflow is insufficient to prevent gas from entering what is ideally the liquid outlet. Hence, a CSD-C operating in such a mode serves to create a fully gas overflow and a two phase underflow (which is indeed the intended experimental operation in the cascade mode).

The second of the fully inhomogeneous modes is that of proper core flow (Fig. 5b). In this flow state an axial gas core is formed such that the baffle plate is able to accommodate the size of the liquid annulus. The core can exist in a stable circular cylindrical form (much like the gas core in terrestrial separators ${ }^{20}$ ) provided that the surface instabilities and body forces are small compared to the centripetal acceleration. The stable existence of the core is limited by unaccounted capillary effects, but the Weber number of the gas core for the majority of the flow rates considered generally lay beyond what has been established as a rough criterion for stability. ${ }^{5}$

The last of the modes of operation is that of the collapsed core (Fig. 5c). The fluids in this state are initially separated by the radial pressure gradients, but the respective liquid and gas flow rates are in such a ratio so as to allow fouling of the overflow by the liquid stream. For the baseline CSD-C design, this collapsed core is found to occur (not surprisingly) when the liquid volumetric quality becomes high compared to the gas quality. This, however, is by no means a general feature of cyclonic separator designs. In fact, simple modifications to the separator geometry can allow collapsed core conditions to exist for arbitrarily high gas volumetric qualities ( 0.9 and beyond) or allow proper gas cores to persist under the conditions of pure liquid injection.

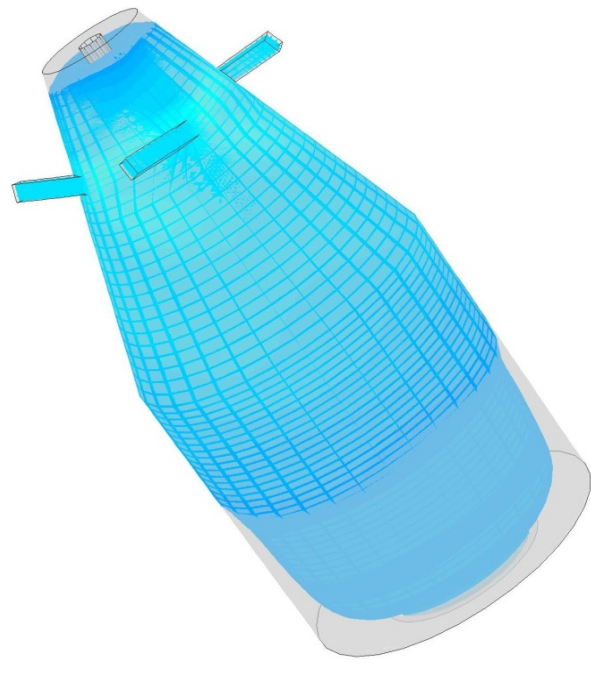

Figure 4. Semi-inhomogeneous Operation (visualization by Filled Gas Volume Fraction Contours) 


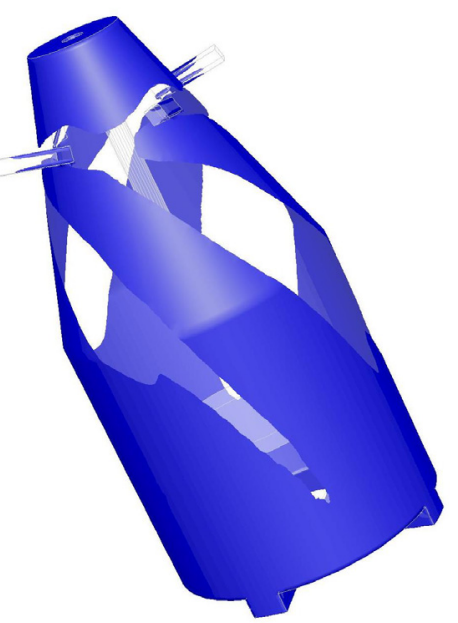

(a)

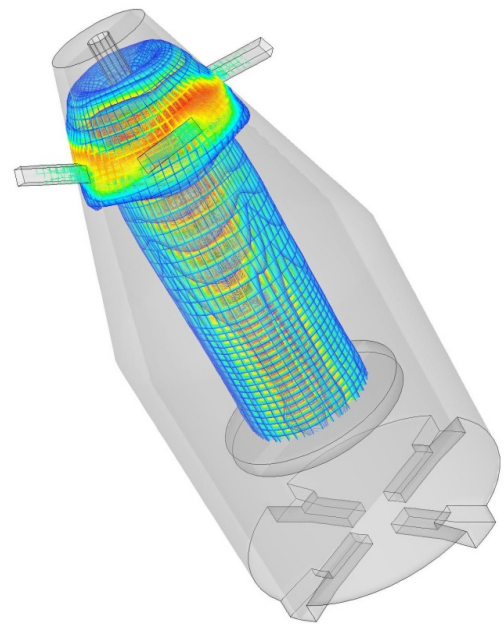

(b)

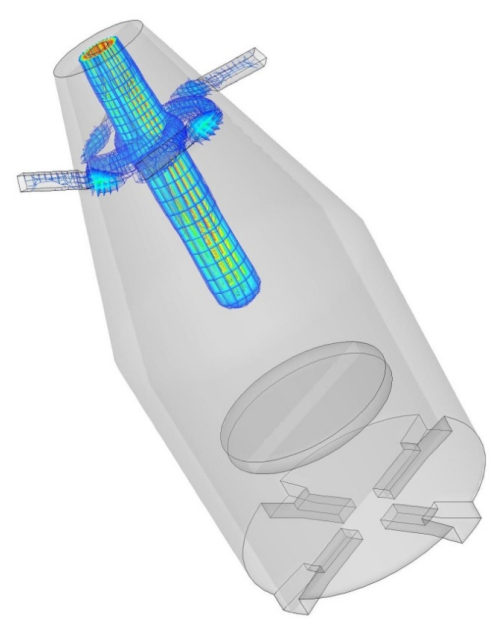

(c)

Figure 5. (a) Adhered film Visualization by Clipped Wall Iso-Surface of Gas Volume Fraction, (b) Core Proper Visualization by Filled Gas Volume Fraction Contours, (c) Collapsed Core Visualization by Filled Gas Volume Fraction Contours

\section{Performance Metrics}

As the injection of the two phase mixture occurs at a relatively small radius, and, furthermore, as the nominal flow conditions are of high gas volumetric quality, the separation process is typically able to fully divide the flow into pure media (at least initially) in the baseline cyclonic separator. The performance of the CSD-C when operating in such inhomogeneous modes was fully investigated by examining the flow characteristics that arise across the range of volumetric qualities for two differing separation-assured total volumetric flow rates.

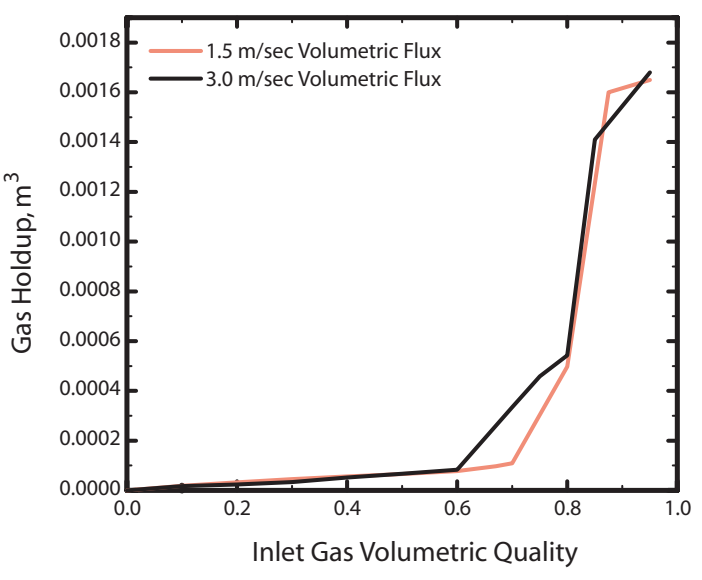

Figure 6. Core Volume versus Inlet Volumetric Quality for Baseline CSD-C

Table 3. Inhomogeneous Mode Classification for the Baseline CSD-C

\begin{tabular}{lcc}
\hline \hline & \multicolumn{2}{c}{ Range of Quality } \\
& $9.7 \mathrm{~L} / \mathrm{min}$ & $19.4 \mathrm{~L} / \mathrm{min}$ \\
\cline { 2 - 3 } Collapsed Core & 0.0 to .7 & 0.0 to .65 \\
Core Proper & .7 to .85 & .65 to .85 \\
Adhered Film & .85 to 1.0 & .85 to 1.0 \\
\hline \hline
\end{tabular}

Figure 6 shows the variation as such of the gas holdup with respect to the gas volumetric quality for the two large volumetric flow rates of $9.7 \mathrm{~L} / \mathrm{min}$ and 19.4 $\mathrm{L} / \mathrm{min}$ (corresponding respectively to total volumetric fluxes of $1.5 \mathrm{~m} / \mathrm{s}$ and $3.0 \mathrm{~m} / \mathrm{s}$ ). The gas holdup represents the total volume of within the separator that is occupied by the gas phase.

The wide variability of the gas holdup values arises as the separator passes from one of its inhomogeneous modes of operation into another. The modal ranges for the above conditions can be roughly tabulated as in Table 3 . The highly sloped regions correspond to the proper core mode as the expansion of the gas core is able to accommodate the pressure mismatch incurred by differing mass flow rates through the respective outlets.

The efficacy of the separator is extremely sensitive to its mode of operation. In analogy to the flow split parameter from terrestrial hydrocyclones, the underflow separator efficacy and the overflow separator efficacy, can be defined as

$$
\begin{aligned}
& \varepsilon_{\text {underflow }}=\left(A_{\text {underflow }} j_{\text {liquid, underflow }}\right) /\left(A_{\text {inlet }} j_{\text {liquid, inlet }}\right) \\
& \varepsilon_{\text {overflow }}=\left(A_{\text {oveflow }} j_{\text {gas, overflow }}\right) /\left(A_{\text {inlet }} j_{\text {gas, inlet }}\right)
\end{aligned}
$$


Thus, $\varepsilon_{\text {underflow }}$ can be seen as characteristic metric ranging from 0 to 1 that indicates the degree to which the liquid phase is removed through the appropriate underflow outlet. $\varepsilon_{\text {overflow }}$ can be similarly viewed as the degree to which the gas phase is removed through its intended overflow outlet.

Plots of these efficacies were made across the range of volumetric qualities for the same flow rates as before (Fig. 7).
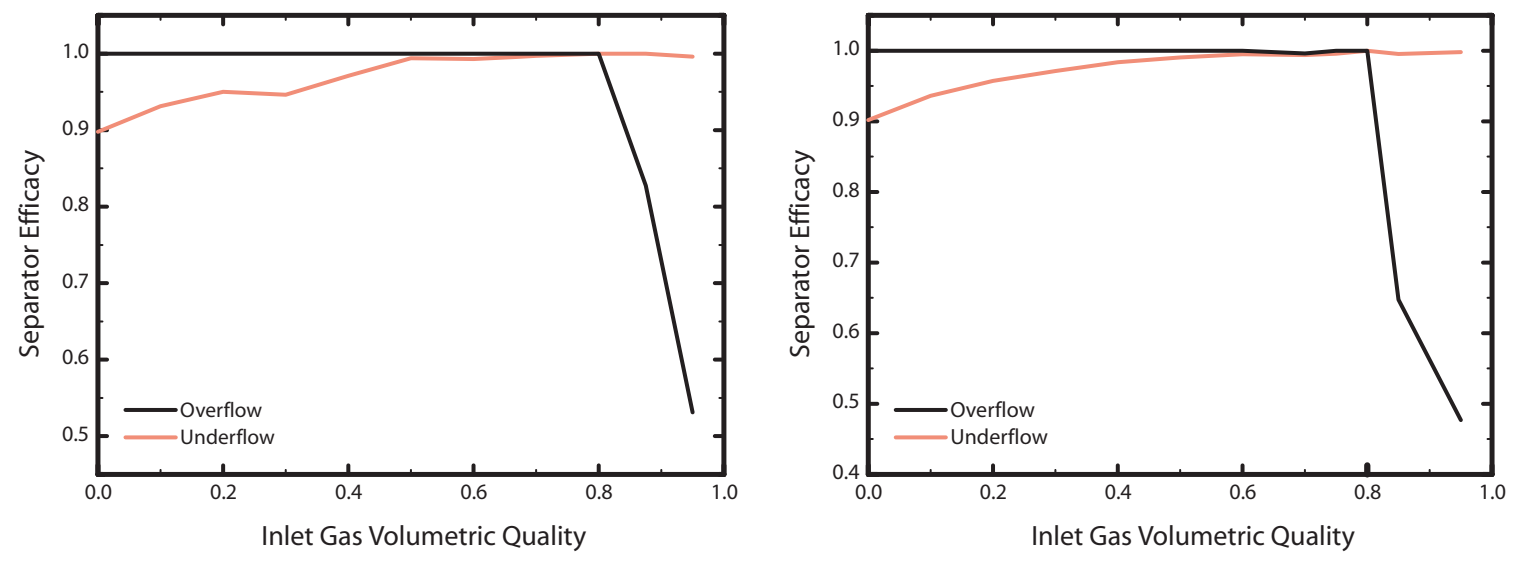

Figure 7. Separator Efficacies for $9.7 \mathrm{~L} / \mathrm{min}$ (left) and $19.4 \mathrm{~L} / \mathrm{min}$ (right) Combined Injection into Baseline CSD-C

These similar plots indicate the respective performance of the individual modes. The core collapse mode can be seen to cause a drop in $\varepsilon_{\text {underflow }}$ by virtue of liquid encroachment of the overflow. Similarly, the adhered film mode dictates a greatly reduced $\varepsilon_{\text {overflow }}$ by virtue of gas encroachment of the underflow. The proper core and collapse/proper core transition modes however operate (according to these initial calculations) with nearly perfect separation efficacies at both outlets. The range of complete separation is predicted to be $0.5<\beta_{\text {gas }}<0.8$ for the 19.4 $\mathrm{L} /$ min consideration and $0.6<\beta_{\text {gas }}<0.8$ for the $9.7 \mathrm{~L} / \mathrm{min}$ consideration.

These predictions of nearly perfect separation must be approached carefully, however. Considering the injected fluid to be comprised of some single typical Sauter bubble/droplet diameter can clearly misleadingly predict total separation of the fluid components. While the momentum interchange of a complete distribution of bubble sizes en mass acts similarly by construction of the Sauter diameter specification, the individual bubbles would respond differently to the separation pressure gradients. Thus, small distributed bubbles could act partly in a homogeneous manner thereby reducing the gas outlet efficacy. The fouling of the underflow in this manner should, however, prove to be largely negligible as the Sauter mean bubble diameter chosen for the above simulations (and for most subsequent simulations) was an already fairly small $1 \mathrm{~mm}$.

\section{Phenomenological Description and Device Optimization}

The issue of pressure matching mechanisms to achieve a common gage at the respective outlets turns out to be the primary factor dictating the modal operation of the separator. The mechanisms available to the flow to induce the appropriate pressure drop to achieve open atmospheric conditions at the pressure boundaries include both flow augmentation at the outlets and the pressure gradients afforded by the cyclonic flow itself. The interplay of these respective mechanisms determines the achieved mode. This process is roughly analogous to the interplay that determines the liquid level in a terrestrial gas-liquid separator. ${ }^{21}$

Specifically, beginning with the adhered film mode, the pressure drop induced by the high volumetric flow of the gas creates a significant pressure loss at the overflow outlet. No such pressure drop can be generated by losses of the liquid phase alone entering the underflow as the respective flow rates are very much lower (for the typical high gas quality where adhered films are generated for the baseline design). Hence, pressure matching requires the augmentation of the underflow by encroachment of some fraction of the gas flow.

As the gas quality at the inlet is reduced, the pressure drop created by the gas at the overflow is diminished while the drop created by the liquid at the underflow is simultaneously increased. At converse combinations of flow rates a situation similar to that described above arises whereby the gas can now no longer support the necessary pressure drop at the overflow. Instead of enforcing this disparity with upward fouling by the liquid phase, the pressure drop can instead be facilitated by the rotation of the flow. The proper gas core thus acts as a flow 


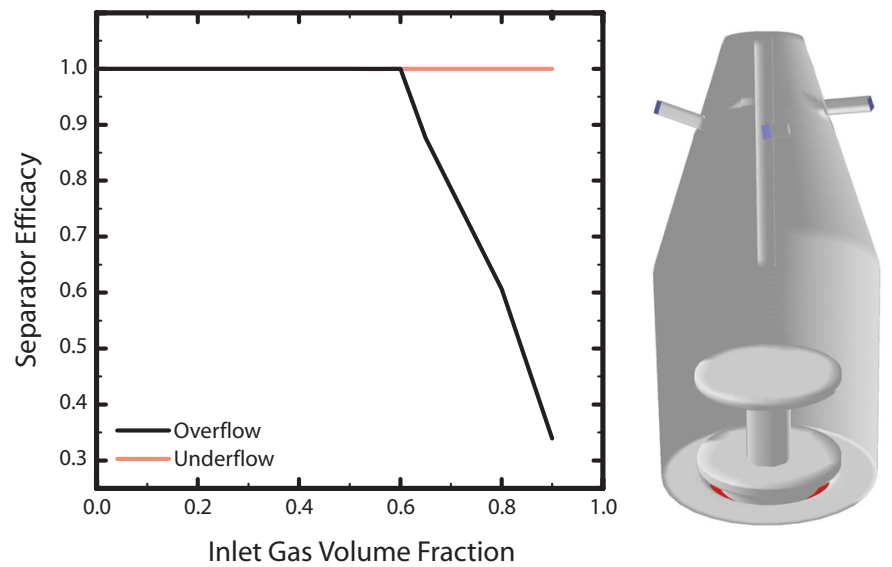

Figure 8. Separator Efficacy versus Inlet Gas Volumetric Quality for Modified CSD-C (right)

moderation mechanism that can expand or contract to provide the exact pressure drop across the overflow outlet such that the outward mass flow rate assumes its correct value as dictated by continuity. This mechanism is stable in that an increase in the gas mass flow rate can be imagined to cause an instantaneous increase in the gas core radius. This increase in the radius thereby reduces the total pressure drop facilitated by the vortex which in turn forces the overflow to accommodate a higher pressure drop. The overflow can only do so by increasing the gas flow rate outward, and the situation is therefore stable. The stability, however, is limited by the relative total pressure drop that can be accommodated by the swirl as compared to that of the outlets. Beyond the limits of this vortex core generated pressure drop, the pressure matching can only be maintained by encroachment of the liquid stream to the overflow, as demonstrated by the predicted collapsed core mode of operation.

The very narrow range of core proper operation for the baseline CSD-C design indicates that it is almost entirely outlet mediated as the gas core is unable to support any wide mismatch in pressures. Changes in the flow injection rates do not tend to change the behavior particular to the baseline geometry as all of the associated pressure drop mechanisms are proportional to roughly the square of the characteristic velocity in the flow (i.e. the radial pressure gradient in the vortex is related to the tangential velocities squared while the outlet pressure drop is a minor loss that is too roughly velocity-squared dependent). The biased behavior is not surprising as the device was designed for a cascade mode of high gas volumetric flow rates. Modification of the outlets, however, is enough to create a wide range of tailored performance characteristics as the device can be made to be outlet mediated or core mediated depending upon the desired design properties.

An example of one of many such optimizations, shown in Fig. 8, allows the core to remain stable for continuous liquid-only injection (as demonstrated by the efficacy map). Such a design is suited to the task of the separation of a dispersed gaseous two-phase injection where the purity of the overflow is tantamount. The purity of the underflow, conversely, is sacrificed in this design (particularly for low total volumetric fluxes). Any of these particular modifications, however, is dependent upon the specified gage pressure at the respective outlets, and, hence, would necessarily need to be approached at a systems level for any inclusion in a complete flow system.

\section{Conclusion}

Gas-liquid separation is an important multiphase flow issue that arises in the absence of gravity. The vortex flows within one such family of devices, the cyclonic gas-liquid microgravity separators, are quite complicated and represent a considerable challenge to any attempted modeling effort. The work completed thus far has generated a reasonably solid foundation upon which to base any future computational fluid dynamics investigation. While multiple deficiencies in the methodologies therein do exist inherent to the framework that has been developed, the respective regions of CFD applicability and questionability have been delimited as well as is possible.

Although the present study has produced logically consistent results regarding the performance characteristics of the general CSD-C separator, a great deal of work remains to be completed before its operation can be considered to be exhaustively categorized.

A host of computational fluids dynamics approaches should be explored to address the deficiencies of the models currently employed. For the film mode of operation, issues such as wall adhesion and full surface tension resolution at the liquid-gas interface must be fully examined. To do so, a VOF/Euler-Euler hybrid method would likely have to be pursued to capture all of the salient features of the flow. Surface features must too be captured for the analysis of the core proper mode so as to address the capillary disruption with more rigor than the simple demarcation of a Weber number failure criterion. A method to account for bubble coalescence and break-up (and, symmetrically speaking, droplet fission and fusion) must too be explored as these phenomena are important given the amount of gas holdup that is often entrained within the separator volume. 
In general, many of the modeling choices (such as the specified interphase momentum coefficients and the turbulence modification therein) are in desperate need of experimental validation which can only be generated by a significant device-level microgravity investigation. Steady state itself should be moved beyond in any future computational study as the issues of startup and unsteady injection (including both small temporal scale slug flow considerations and device level time variance) could have a significant effect upon the operation of the CSD-C. Issues therein include temporal carry-over and carry-under phenomena. ${ }^{5}$

In spite of all of the above recommendations regarding deficiencies in the current study, it is believed that the insight gained through the current computational study may indeed be successfully employed to create new and better cyclonic separators to meet the future needs of manned and unmanned spaceflight.

\section{Acknowledgments}

This work is supported by the NASA Graduate Student Researchers Program Fellowship. Additional funding was provided by the Case School of Engineering (CSE) Prime Fellowship.

\section{References}

${ }^{1}$ McQuillen, J; Sankovic, J; Hall, N., "Multiphase Flow Separators in Reduced Gravity," Proceedings of IMECE2005, Orlando, $F, 2005$.

${ }^{2}$ Ellis, M.; Best, F.; Kurwitz, C., "Development of a Unique, Passive, Microgravity Vortex Separator," Proceedings of IMECE2005, Orlando, FL, 2005

${ }^{3}$ Ahn, H; Tanaka, K, Tsuge, H., Terasaka, K., Tsukada, K., “Centrifugal gas-liquid separation under low gravity conditions," Separation and Purification Technology 19, pp. 121-129, 2000

${ }^{4}$ McQuillen, J.B., Neumann, E.S., "Two-Phase Flow Research Using the Learjet Apparatus," NASA Technical Memorandum, NASA, Cleveland, OH, TM 106814.pp. 1-14, 1995

${ }^{5}$ Bean, D., Best, F., Waghela, P., Kurwitz, C., "Vortex Necking Phenomena Under Microgravity," Proceedings of the Thermal Fluids Analysis Workshop 2002, NASA Johnson Space Center, 2002

${ }^{6}$ Schrage, D.; Shoemaker, J.; McQuillen, J., "Passive Two-Phase Fluid Separation," Proceedings of the $36^{\text {th }}$ Aerospace Sciences Meeting and Exhibition, Reno, NV, 1998

7 "Microgravity Research in Support of Technologies for the Human Exploration and Development of Space and Planetary Bodies" Commission on Physical Sciences, Mathematics, and Applications," pp. 174-176. National Academies Press. Washington, D.C. 2000

${ }^{8}$ Fluent, et al. (2005). Fluent 6.2 User Guide.

${ }^{9}$ Lane, G.L., Schwarz, M.P., Evans, G.M., "Predicting Gas-Liquid Flow in a Mechanically-Stirred Tank," Second International Conference on CFD in the Minerals and Process Industries, CSIRO, Melbourne, Australia, 1999

${ }^{10}$ Gidaspow , D., Multiphase flow and fluidization : continuum and kinetic theory descriptions. Academic Press. Boston, MA, 1994

${ }^{11}$ Brennan, C. , Fundamentals of Multiphase Flow. Cambridge University Press, New York, NY, 2005

${ }^{12}$ Sanyal, J; Marchiso, D; Fox, R., Dhanasekharan, K., "On the Comparison between Population Balance Models for CFD Simulation of Bubble Columns," Ind. Eng. Chem. Res. 44. pp. 5063-5072, 2005

${ }^{13}$ Wilcox, D. Turbulence Modeling for CFD, Second Edition, DCW Industries, La Canada, CA, 2004

${ }^{14}$ Lane, G.L., Schwarz, M.P., Evans, G.M., "Development of Improved Methods for Computational Modeling of Gas Dispersion in Stirred Tanks," Third International Conference on CFD in the Minerals and Process Industries, CSIRO, Melbourne, Australia., 2003

${ }^{15}$ Bove, Stefano., "Computational Fluid Dynamics of Gas-Liquid Flows Including Bubble Population Balances," Thesis, Doctor of Philosophy, Group for Chemical Fluid Flow Processes, Aalborg University, 2005

${ }^{16}$ C. Colin, J. Fabre, and J. B. McQuillen, "Bubble And Slug Flow At Microgravity Conditions: State Of Knowledge And Open Questions," Chemical Engineering Communications, 141-142, pp. 155-173, 1996

${ }^{17}$ Hsieh, K.T., Rajamani, R.K., "Mathematical Model of the Hydrocyclone Based on Physics of Fluid Flow," AIChE Journal, Vol.37, No.5. pp. 735-746, 1991

${ }^{18}$ Slack, M. D., Del Porte, S., Engelman, M.S., "Designing Automated Computational Fluid Dynamics Modeling Tools for Hydrocyclone Design," Enductive Systems Inc. Technical Memorandum. Retrieved from the World Wide Web: http://www.enductive.com/pdf/paper_hydrocyc_2003.pdf

${ }^{19}$ Hoyt, Nathaniel, "Computational Investigation of the NASA Cyclonic Separation Device," Masters Thesis, Department of Mechanical and Aerospace Engineering, Case Western Reserve University, Cleveland, OH, 2007

${ }^{20}$ Monredon, T.C., Hsieh, K.T. and Rajamani, R.K., "Fluid flow model of the hydrocyclone: an investigation of device dimensions," International Journal of Mineral Processing, Vol.35. pp. 65-83.

${ }^{21}$ Movafaghian, S., Jaua-Marturet, J.A., Mohan, R.S., Shohoam, O., Koubam G.E., "The effects of geometry, fluid properties and pressure on the hydrodynamics of gas-liquid cylindrical cyclone separators," International Journal of Multiphase Flow. Vol 26. pp. 999-1018 